\title{
Frequent KRAS mutation in complex mucinous epithelial lesions of the endometrium
}

\author{
Ahmed Alomari, Rita Abi-Raad, Natalia Buza and Pei Hui \\ Department of Pathology, Yale University School of Medicine, New Haven, CT, USA
}

\begin{abstract}
KRAS mutation correlates with mucinous differentiation in various human cancers, and recently, was found in a high proportion of a small cohort of papillary mucinous lesions of the endometrium. In this study, a large number of endometrial mucinous lesions were analyzed for the presence of KRAS mutation along with clinical progression. A total of $\mathbf{4 5}$ endometrial biopsy/curettage cases were included in the study and classified into the following categories: simple mucinous change (5 cases), complex mucinous change (33 cases) and mucinous adenocarcinoma (7 cases). Follow-up hysterectomy specimens were available in 14 of 33 patients (42\%) with complex mucinous lesions, of which 9 cases $(64 \%)$ showed atypical complex hyperplasia with an average interval of 21 weeks. None of the 5 cases of simple mucinous change showed KRAS mutation. KRAS mutation was observed in 18 of 33 patients with complex mucinous lesions (55\%) and in 6 of 7 cases of mucinous adenocarcinoma (86\%). Overall, KRAS mutation has a positive predictive value (PPV) of $88 \%$ (7/8 cases) for complex atypical hyperplasia or adenocarcinoma in the follow-up hysterectomy. In conclusion, the current data further emphasizes the architectural complexity as an important prognostic indicator for patients with mucinous endometrial lesions. The presence of KRAS mutation in both mucinous adenocarcinoma and complex mucinous changes indicates that KRAS mutational activation is implicated in the pathogenesis of a significant subset of endometrial mucinous carcinoma. With a high PPV, KRAS mutation analysis may offer an additional discriminatory power to refine risk stratification algorithm for patients with endometrial mucinous lesions. Modern Pathology (2014) 27, 675-680; doi:10.1038/modpathol.2013.186; published online 1 November 2013
\end{abstract}

Keywords: endometrial; KRAS; mucinous lesion

Endometrial epithelial metaplasia refers to a morphologically heterogeneous group of proliferations and differentiations that lead to partial or complete replacement of the endometrial epithelium with another type. ${ }^{1,2}$ Mucinous metaplasia, including both the typical endocervical and the rare intestinal types, is particularly relevant as it is not infrequently encountered in an endometrial biopsy or curettage specimen of menopausal or postmenopausal patients and is more likely associated with additional aggressive endometrial lesions. However, the frequent disparity between the cytological atypia and architectural alterations in a mucinous lesion, especially in a small, fragmented biopsy may lead to significant diagnostic challenges of interpretation to guide patient management. ${ }^{2,3}$ Type I endometrial

Correspondence: Dr P Hui, MD, PhD, Department of Pathology, Yale University School of Medicine, BML254B, 310 Cedar Street, New Haven, CT 06520-8023, USA.

E-mail: pei.hui@yale.edu

Received 11 May 2013; revised 20 August 2013; accepted 21 August 2013; published online 1 November 2013 adenocarcinomas frequently show mucinous differentiation, a subset of which are classified as mucinous adenocarcinoma when the tumor contains $50 \%$ or more mucinous component. The latter diagnosis also includes the low-grade mucinous adenocarcinoma ${ }^{4}$ and microglandular variant of endometrioid adenocarcinoma. ${ }^{5-7}$ It has been hypothesized that subtypes of endometrial mucinous metaplasia are biologically related to endometrial adenocarcinoma as precursor lesions, and different classification systems have been proposed based solely on morphological grounds to predict the risk of subsequent endometrial cancer, ${ }^{2,3,8,9}$ particularly mucinous adenocarcinoma. ${ }^{10}$ Endometrial mucinous lesions have been recently proposed to be classified into simple and papillary/complex groups, based on the presence or absence of intraglandular papillary tufts or complex glandular architecture. The lesions within the latter group showed a high incidence of KRAS mutation in association with overexpression of P16, PAX2 and PR, suggesting that they may be precursor lesions of mucinous endometrial adenocarcinoma. ${ }^{11}$ 
KRAS is a key oncogene in the EGFR signaling cascade (RAS-MAPK pathway) and its mutation is an early oncogenic event in the development of human cancers. ${ }^{12}$ Nearly all KRAS mutations occur in codon 12 or 13 of exon 2 of the gene leading to a mutant oncoprotein that is locked in its activation conformation, resulting in constant activation of the signaling pathway in the absence of ligand activation. ${ }^{13}$ It is of particular interest that the presence of KRAS mutation correlates with mucinous differentiation in cancers of various human organs including pancreas, colon and lung. ${ }^{12}$ KRAS is frequently mutated in ovarian mucinous neoplasms and endometrial mucinous carcinoma as well. ${ }^{14}$ Prompted by the recent report of a high frequency of KRAS mutations found in a small cohort of endometrial papillary mucinous lesions, ${ }^{11}$ we investigated various mucinous lesions for the presence of KRAS mutation in endometrial biopsy or curettage specimens in correlation with clinical progression.

\section{Materials and methods}

\section{Case Selection and Histological Assessment}

A retrospective text search for endometrial biopsy or curettage specimens with a final diagnosis of endometrial mucinous change or metaplasia and mucinous carcinoma was performed in the pathology archives from 1983 to 2011 at a single tertiary medical center. The original H\&E. slides of each case were reviewed microscopically to confirm the presence of endometrial mucinous lesions. Similar to previous studies emphasizing the importance of structural complexity and cytological atypia in mucinous changes, ${ }^{3,11}$ the cases were classified into the following categories: simple mucinous change, complex mucinous change and mucinous adenocarcinoma. Simple mucinous change was defined by the presence of linear or pseudostratified epithelial lining with minimal architectural complexity (mild epithelial tufting was permitted) and no or minimal cytological atypia (Figure 1a). Complex mucinous changes included the presence of mucinous epithelium or glands with epithelial papillation, stratification, presence of microglandular or cribriforming configuration (Figures $1 \mathrm{~b}$ and $\mathrm{c}$ ). Focal mild-to-moderate cytological atypia was allowed including variably enlarged nuclei, pseudostratification and presence of prominent nucleoli (Figure 1d). Rare cases with moderate cytological atypia in an otherwise simple mucinous lesion were classified into the complex mucinous category. ${ }^{3}$ However, cases with significant suspicion for carcinoma-due to the presence of more complex architectural changes (extensive papillation or cribriforming) and/or severe cytological atypia-were excluded from the complex mucinous category and classified as adenocarcinoma in this study. Mucinous adenocarcinoma was qualified by the presence of intracytoplasmic mucin production in at least $50 \%$ of tumor cells within a type I endometrial carcinoma (Figures $1 \mathrm{~d}$ and f). The patients' demographics and additional follow-up specimens (whenever available) were reviewed. Hysterectomy with examination of the entire endometrium was considered as the end point of the follow-up.

\section{KRAS Mutation Analysis by Polymerase Chain Reaction-Single Strand Conformation Polymorphism}

With corresponding $\mathrm{H} \& \mathrm{E}$ section to ensure the presence of lesional tissue, $5 \mu \mathrm{m}$ sections of formalin-fixed, paraffin-embedded tissue blocks of each case were prepared for KRAS mutation analysis as described previously. ${ }^{15}$ Briefly, DNA was extracted from unstained tissue sections using Qiagen tissue kit according to the manufacturer's instruction (Qiagen tissue kit, Qiagen, Chatsworth, CA, USA). Exon 2 of the KRAS gene was amplified by polymerase chain reaction (PCR) using flanking primers: forward, 5'-GACTGAATATAAACTTGTG G-3' and reverse, 5'-CTGTATCAAAGAATGGTCC $\mathrm{T}-3^{\prime}$ in a $50 \mu \mathrm{l}$ PCR reaction solution containing $1 \times$ PCR buffer, $0.1 \mathrm{mM}$ dNTP, $1.5 \mathrm{mM} \mathrm{MgCl}_{2}$ and $2.5 \mathrm{U}$ of AmpliTaq Gold DNA polymerase. PCR started with initial denaturation at $95^{\circ} \mathrm{C}$ for $8 \mathrm{~min}$, followed by 35 cycles of denaturation at $94{ }^{\circ} \mathrm{C}$ for $1 \mathrm{~min}$, annealing at $55^{\circ} \mathrm{C}$ for $1 \mathrm{~min}$ and synthesis at $72{ }^{\circ} \mathrm{C}$ for $2 \mathrm{~min}$, and finished by a final extension at $72{ }^{\circ} \mathrm{C}$ for $10 \mathrm{~min}$ (ABI Veriti Thermal Cycler; Applied Biosystem, Foster City, CA, USA). The PCR product was analyzed by single strand conformation polymorphism (SSCP) using $4 \mu \mathrm{l}$ of the PCR product on MDE non-denaturing gel. Electrophoresis was carried out on ice for $2 \mathrm{~h}$ and $45 \mathrm{~min}$ at $325 \mathrm{~V}$. The SSCP gel was then stained with SYBR Gold (Molecular Probes) 1:10 000 in TE buffer added for $20 \mathrm{~min}$ and imaged by Bio-Rad GelDoc UV System (Bio-Rad, Hercules, CA, USA). The presence of KRAS mutation was determined by comparing the SSCP banding patterns with those of five positive controls with known KRAS mutations (Figure 2).

\section{Results}

Forty-five cases of endometrial mucinous lesions were included in this study based on their histological findings meeting the defined criteria (see Materials and methods) and an informative KRAS mutation status. The age of the patients ranged from 44 to 86 years with a mean of 63 years and median of 61 years. Among the 42 patients with available clinical information, $37(80 \%)$ were menopausal or postmenopausal and presented with uterine bleeding at the time of endometrial biopsy or curettage. Based on the defined morphological criteria, the cases were classified into simple mucinous change 

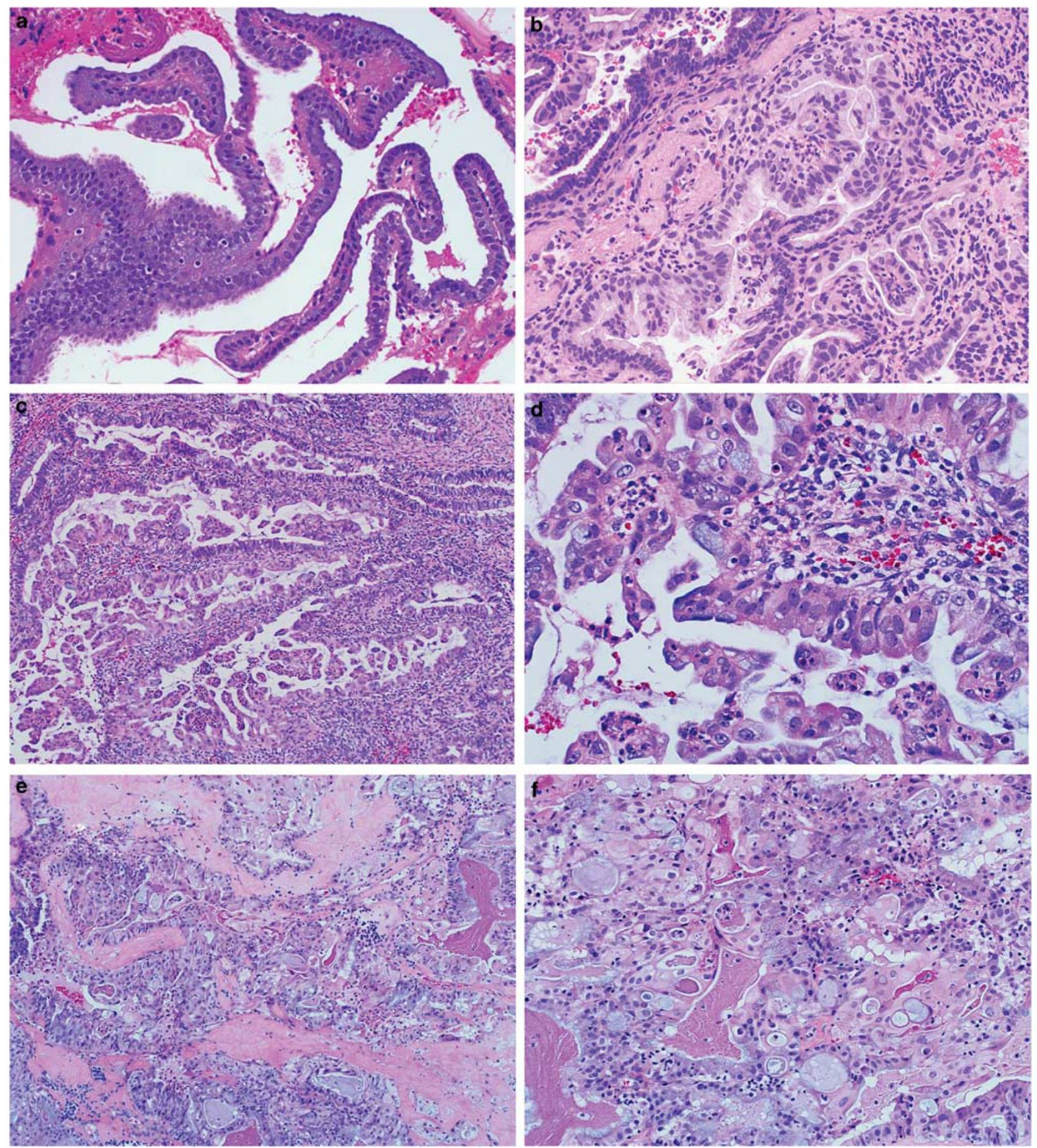

Figure 1 Histological classifications of endometrial mucinous lesions: simple mucinous changes (a), complex mucinous changes with variable degrees of papillation (b and $\mathbf{c}, \mathrm{H} \& \mathrm{E} \times 10)$ and mild-to-moderate cytological atypia $(\mathbf{d}, \mathrm{H} \& \mathrm{E} \times 20)$ and mucinous adenocarcinoma $(\mathbf{e}, \mathrm{H} \& \mathrm{E} \times 10$ and $\mathbf{f}, \mathrm{H} \& \mathrm{E} \times 20)$.

(5 cases), complex mucinous change (33 cases) and mucinous adenocarcinomas (7 cases), with an average age of 61,60 and 75 years, respectively (Table 1).

Thirty-six cases had follow-up specimen(s) with an average follow-up interval of 35weeks (range: 2-145 weeks), including follow-up biopsy or curettage in 14 cases and hysterectomy specimen in 22 cases. Two of five patients with simple mucinous change had a follow-up curettage or hysterectomy after an average of 86 weeks that showed benign endometrium (one was atrophic and the other one was inactive endometrium). Followup hysterectomy specimens (Table 2) were available 
in 14 of 33 patients ( $42 \%)$ with complex mucinous lesions, 9 of which (64\%) showed atypical complex hyperplasia (7 cases) or adenocarcinoma (2 cases) with an average interval of 21 weeks (range: 8-55 weeks). Benign endometrial findings (atrophic or inactive endometrium, endometrial polyp or nonatypical simple hyperplasia) were found in the remaining five cases of complex mucinous lesion in their follow-up hysterectomies after an average interval of 31 weeks (range: 6-90 weeks).

None of the five cases with simple mucinous change showed KRAS mutation. KRAS mutation was observed in 18 of 33 patients with complex mucinous lesions (55\%) (Figure 2 and Table 2). Overall, in cases with a follow-up hysterectomy, KRAS mutation had a positive predictive value (PPV) of $88 \%$ (7/8 cases) and a negative predictive value of $67 \%$ (4/6) for complex atypical hyperplasia or carcinoma. When considering both hysterectomy and curettage as the end point of follow-up, the PPV of KRAS mutation decreased to $67 \%$ (10 of 15 cases)



Figure 2 KRAS mutation analysis by polymerase chain reactionsingle strand conformation polymorphism (PCR-SSCP) showing mutation banding patterns found in representative complex mucinous lesions (Case 1: GAT mutation shown in duplicates; Case 2: TGT mutation shown in duplicates). The wild-type KRAS is GGT. Known positive controls show distinct banding patterns (GTT, AGT, GAT, GCT and TGT).

Table 1 Histological subtypes of endometrial mucinous lesions and $K R A S$ mutation $(N=45)$

\begin{tabular}{llccc}
\hline $\begin{array}{l}\text { Total } \\
\text { no. }\end{array}$ & $\begin{array}{l}\text { Mucinous } \\
\text { lesion subtype }\end{array}$ & $\begin{array}{c}\text { Average age } \\
\text { (years) }\end{array}$ & $\begin{array}{c}\text { KRAS } \\
\text { mutation }\end{array}$ & $\begin{array}{c}\text { \% KRAS } \\
\text { mutation }\end{array}$ \\
\hline 5 & Simple & 61 & 0 & 0 \\
33 & Complex & 60 & 18 & 55 \\
7 & Mucinous & 75 & 6 & 86 \\
& carcinoma & & & \\
\hline
\end{tabular}

in predicting complex atypical hyperplasia or carcinoma. Among the patients with complex mucinous lesions with a wild-type KRAS gene, $42 \%(5 / 12$ cases $)$ had a diagnosis of complex atypical hyperplasia or carcinoma in their followup curettage or hysterectomy specimens. KRAS mutation was found in six of seven patients $(86 \%)$ (Table 2) with a biopsy diagnosis of mucinous adenocarcinoma and all patients had FIGO stage 1 or 2 adenocarcinoma in their subsequent staging hysterectomy.

\section{Discussion}

Endometrial epithelial metaplasias are traditionally classified on the morphological ground into squamous, mucinous, papillary syncytial, ciliated/ tubal, eosinophilic/oxyphilic, clear-cell and hobnail cell subtypes. They commonly coexist in the same biopsy specimen ${ }^{3,8}$ and frequently are associated with pathologic conditions including polyps, endometritis, hyperplasia and adenocarcinoma. ${ }^{1-3,16-18}$ Mucinous change represents approximately $24 \%$ of endometrial metaplasia and is characterized by the presence of epithelial cells that have a distinctive watery blue appearance (endocervical-type epithelium) as a result of cytoplasmic accumulation of PAS-positive diastaseresistant mucin material. ${ }^{1}$ Very rarely, mucinous changes of the endometrium exhibit intestinal-type differentiation with cellular brush border, goblet cells and neuroendocrine cells, although this is more common in the cervix where it is frequently associated with an endocervical neoplastic process. ${ }^{2}$ In fact, intestinal differentiation was not observed in any of the endometrial mucinous lesions in this study. The architectural patterns of mucinous metaplasia vary widely from simple epithelial pattern to variable degrees of complexity including epithelial branching, budding, papillation, cribriforming or microglandular formation. Moreover, disparity between architectural complexity and cytological atypia in mucinous lesions frequently exists and therefore poses a diagnostic challenge, particularly when dealing with a small biopsy or curettage specimen. ${ }^{2,3}$

The frequent association and shared common pathways between endometrial metaplastic changes and neoplasia stress the importance of studying

Table 2 KRAS mutation in correlation with complex atypical hyperplasia (CAH) or adenocarcinoma (CA) present in follow-up hysterectomy $(N=22)$

\begin{tabular}{|c|c|c|c|c|c|}
\hline \multirow[b]{2}{*}{ Total no. } & \multirow[b]{2}{*}{ Mucinous lesion subtype } & \multicolumn{2}{|c|}{ KRAS positive } & \multicolumn{2}{|c|}{ KRAS negative } \\
\hline & & No. $(\%)$ & $C A H$ or $C A(\%)$ & No. $(\%)$ & $C A H$ or $C A(\%)$ \\
\hline 1 & Simple & $0(0)$ & $0(0)$ & $1(100)$ & $0(0)$ \\
\hline 14 & Complex & $8(57)$ & 7 (1 CA and 6 CAH) (88) & $6(42.8)$ & 2 (1 CA and $1 \mathrm{CAH})(33)$ \\
\hline 7 & Mucinous Carcinoma & $6(86)$ & $6(100)$ & $1(14)$ & $1(100)$ \\
\hline
\end{tabular}


metaplasias as potential precursors for endometrial epithelial malignancies. ${ }^{19-22}$ The risk of associated malignancy in mucinous changes has been reported significant $^{2}$ and lesions with complex architectural patterns may carry the same prognostic significance as conventional atypical endometrial hyperplasia. ${ }^{23,24}$ It has been proposed that mucinous metaplasia may represent a clonal alteration of endometrial glandular epithelium. ${ }^{25}$ Histological classifications of mucinous metaplasia were attempted in the past to predict subsequent risk of developing endometrial malignancy by various cytological and histological parameters. ${ }^{1,3,8,23}$ Patients with simple architectural patterns of mucinous changes and bland cytological features in the absence of coexisting premalignant change in the non-mucinous epithelium are benign. In contrast, patients with complex mucinous changes have a significant risk of having atypical hyperplasia or adenocarcinoma upon follow-up evaluation with percentages ranging from 30 to $100 \% .^{3,8}$ Mucinous lesions in our current study were classified into two categories: simple and complex. We did not further subclassify complex mucinous lesions, as recent immunohistochemical and molecular genetic investigations indicated that complex mucinous lesions with varying complexity and cytological abnormalities share similar oncogenic profiles as possible precancerous lesions at least for a subset of endometrial adenocarcinomas. ${ }^{11}$ In our study, 54\% (14/35) of patients with complex mucinous changes were found to have complex atypical hyperplasia or adenocarcinoma in their follow-up curettage or hysterectomy specimens. In a study by Nucci et $a l,{ }^{3}$ the majority of complex mucinous lesions were found to have endometrial carcinoma in the follow-up hysterectomy. However, such high frequency of subsequent malignancy was not seen in this current investigation and in others. ${ }^{11}$ The reason for this disparity is unclear but possible explanations include varying histological criteria for case inclusion in different studies, ${ }^{8,11}$ for instance, endometrial mucinous lesions with more extensive architectural complexity and/or severe cytological atypia leading to a significant suspicion for carcinoma were excluded from the complex mucinous category and classified as carcinoma in our study.

KRAS mutation has been linked to epithelial tumors with mucinous differentiation of various organs including pancreas, colon and lung. ${ }^{12}$ The gene is also frequently mutated in ovarian mucinous neoplasms and endometrial mucinous carcinoma. ${ }^{14}$ More recently, KRAS mutation was found in a high proportion of a small, highly selected cohort of complex, papillary mucinous lesions of the endometrium. ${ }^{11}$ Similar to the latter, the current study also identifies a significant difference in the prevalence of KRAS mutation between simple and complex mucinous lesions ( $0 \%$ vs 55\%). Patients with KRAS mutation had a PPV of $88 \%$ (7 of 8 cases) of having atypical complex hyperplasia or carcinoma in their follow-up hysterectomy specimens, although this value dropped to $67 \%$ (10 of 15 cases) if all types of follow-up specimens including curettage and hysterectomy were considered. This likely represents an incomplete evaluation of the endometrium by curetting. Our study also confirms high prevalence $(86 \%)$ of KRAS mutation in endometrial mucinous adenocarcinoma, as reported previously. ${ }^{11}$ High prevalence of KRAS mutation in both complex mucinous lesions and mucinous endometrial adenocarcinoma suggests a related biological progression, a signature pathogenesis implied in mucinous tumors in other human organs. However, the existence of KRAS mutationnegative cases of both endometrial mucinous adenocarcinoma and complex mucinous lesions indicates that KRAS mutational activation is implicated in the pathogenesis of a significant subset but not all cases of endometrial mucinous carcinoma.

In conclusion, the outcome of this study further emphasizes the architectural complexity as an important prognostic indicator for patients with mucinous endometrial lesions. Diagnostic separation of endometrial mucinous metaplasia into morphologically simple and complex categories constitutes a highly sensitive, although not specific, approach to predict the risk of atypical complex hyperplasia or carcinoma of the endometrium. With a high PPV, the presence of KRAS mutation offers an additional discriminatory power in the workup of endometrial mucinous lesions that may progress to or coexist with atypical hyperplasia or adenocarcinoma. Additional studies are important for development of a risk stratification algorithm for patient management.

\section{Disclosure/conflict of interest}

The authors declare no conflict of interest.

\section{References}

1 Hendrickson MR, Kempson RL. Endometrial epithelial metaplasias: proliferations frequently misdiagnosed as adenocarcinoma. Report of 89 cases and proposed classification. Am J Surg Pathol 1980;4:525-542.

2 Nicolae A, Preda O, Nogales FF. Endometrial metaplasias and reactive changes: a spectrum of altered differentiation. J Clin Pathol 2011;64:97-106.

3 Nucci MR, Prasad CJ, Crum CP, et al. Mucinous endometrial epithelial proliferations: a morphologic spectrum of changes with diverse clinical significance. Mod Pathol 1999;12:1137-1142.

4 Fujiwara M, Longacre TA. Low-grade mucinous adenocarcinoma of the uterine corpus: a rare and deceptively bland form of endometrial carcinoma. Am J Surg Pathol 2011;35:537-544. 
5 Young RH, Scully RE. Uterine carcinomas simulating microglandular hyperplasia. A report of six cases. Am J Surg Pathol 1992;16:1092-1097.

6 Zaloudek C, Hayashi GM, Ryan IP, et al. Microglandular adenocarcinoma of the endometrium: a form of mucinous adenocarcinoma that may be confused with microglandular hyperplasia of the cervix. Int J Gynecol Pathol 1997;16:52-59.

7 Giordano G, D’Adda T, Gnetti L, et al. Endometrial mucinous microglandular adenocarcinoma: morphologic, immunohistochemical features, and emphasis in the human papillomavirus status. Int J Gynecol Pathol 2006;25:77-82.

8 Vang R, Tavassoli FA. Proliferative mucinous lesions of the endometrium: analysis of existing criteria for diagnosing carcinoma in biopsies and curettings. Int J Surg Pathol 2003;11:261-270.

9 McKenney JK, Longacre TA. Low-grade endometrial adenocarcinoma: a diagnostic algorithm for distinguishing atypical endometrial hyperplasia and other benign (and malignant) mimics. Adv Anat Pathol 2009; 16:1-22.

10 Ross JC, Eifel PJ, Cox RS, et al. Primary mucinous adenocarcinoma of the endometrium. A clinicopathologic and histochemical study. Am J Surg Pathol 1983;7:715-729.

11 Yoo SH, Park BH, Choi J, et al. Papillary mucinous metaplasia of the endometrium as a possible precursor of endometrial mucinous adenocarcinoma. Mod Pathol 2012;25:1496-1507.

12 Bos JL. ras oncogenes in human cancer: a review. Cancer Res 1989;49:4682-4689.

13 Kranenburg O. The KRAS oncogene: past, present, and future. Biochim Biophys Acta 2005;1756:81-82.

14 Lax SF, Kendall B, Tashiro H, et al. The frequency of p53, K-ras mutations, and microsatellite instability differs in uterine endometrioid and serous carcinoma: evidence of distinct molecular genetic pathways. Cancer 2000;88:814-824.

15 Dillon DA, Johnson CC, Topazian MD, et al. The utility of Ki-ras mutation analysis in the cytologic diagnosis of pancreatobiliary neoplasma. Cancer J 2000;6:294-301.

16 Carlson JW, Mutter GL. Endometrial intraepithelial neoplasia is associated with polyps and frequently has metaplastic change. Histopathology 2008;53: 325-332.

17 Fukunaga M, Ushigome S. Epithelial metaplastic changes in ovarian endometriosis. Mod Pathol 1998;11:784-788.

18 Schlesinger C, Kamoi S, Ascher SM, et al. Endometrial polyps: a comparison study of patients receiving tamoxifen with two control groups. Int J Gynecol Pathol 1998;17:302-311.

19 Andersen WA, Taylor PT Jr., Fechner RE, et al. Endometrial metaplasia associated with endometrial adenocarcinoma. Am J Obstet Gynecol 1987;157: 597-604.

$20 \mathrm{Kaku} \mathrm{T,} \mathrm{Tsukamoto} \mathrm{N,} \mathrm{Tsuruchi} \mathrm{N,} \mathrm{et} \mathrm{al.} \mathrm{Endometrial}$ metaplasia associated with endometrial carcinoma. Obstet Gynecol 1992;80:812-816.

21 Dane C, Tatar Z, Dane B, et al. Clinicopathologic analysis: relationship between endometrial carcinoma and uninvolved endometrium. Eur J Gynaecol Oncol 2009;30:71-74.

22 Kaku T, Silverberg SG, Tsukamoto N, et al. Association of endometrial epithelial metaplasias with endometrial carcinoma and hyperplasia in Japanese and American women. Int J Gynecol Pathol 1993;12:297-300.

23 Kurman RJ, Norris HJ. Evaluation of criteria for distinguishing atypical endometrial hyperplasia from well-differentiated carcinoma. Cancer 1982;49: 2547-2559.

24 Longacre TA, Chung MH, Jensen DN, et al. Proposed criteria for the diagnosis of well-differentiated endometrial carcinoma. A diagnostic test for myoinvasion. Am J Surg Pathol 1995;19:371-406.

25 Jovanovic AS, Boynton KA, Mutter GL. Uteri of women with endometrial carcinoma contain a histopathological spectrum of monoclonal putative precancers, some with microsatellite instability. Cancer Res 1996;56: 1917-1921. 